\title{
ANÁLISIS DE LAS HABILIDADES BLANDAS ENCONTRADAS EN RESEÑAS DE HOTELERÍA A TRAVÉS DE METABUSCADORES
}

\author{
Soft skills analysis for online review by using metasearch engines
}

\author{
Tomas Santiago Suarez Pinzón \\ tosuarez@poligran.edu.co \\ Politécnico Grancolombiano \\ Negocios Internacionales \\ Bogotá, Colombia
}

\section{RESUMEN}

Según el Ministerio de Industria, Comercio y Turismo, en el año 2019 Colombia fue visitada por 4.115.120 turistas extranjeros. Esta información genera cuestionamientos sobre la calidad del servicio que se está ofreciendo en el sector servicios, específicamente en el subsector hotelero, para identificar el nivel de satisfacción del usuario mediante la calificación de Booking y la palabra usada en la reseña para definir a esta misma. Para tal fin, este estudio no se enfocó en incluir la infraestructura y volumen de empleados para proveer el servicio al cliente, sino en el área de atracción y selección de los mejores candidatos que cuenten con habilidades blandas, lo que permitirá a los hoteles brindar una experiencia que se califique como 10/10. Se revisaron artículos relacionados con la importancia de las reseñas y comentarios que reflejen la experiencia del huésped y la importancia de las habilidades blandas en el personal hotelero; igualmente, se revisó y analizó el metabuscador Booking, se realizó un análisis de datos con Rstudio y una recopilación de datos con Parsehub de una muestra estadística para tener un acercamiento a los datos y las calificaciones en línea, para identificar la relación entre una buena calificación y el uso de cuatro habilidades blandas, entre las cuales se encuentran: integridad, relación con el cliente, servicio al cliente y trabajo en equipo. Los puntos para mejorar son coordinación entre las áreas para generar un buen clima organizacional, capacitación en servicio al cliente y rediseñar los procesos de selección en cuanto a vincular personas con las competencias blandas requeridas para atender a los visitantes. Según estudios que se relacionarán a continuación, las reseñas web en la actualidad pueden ser uno de los factores definitivos de un turista a la hora de decidirse entre un hotel u otro.

PALABRAS CLAVE:

habilidades blandas, servicio, trabajo en equipo, cliente, comentarios Booking, Booking 


\section{INTRODUCCIÓN}

El turismo se ha convertido en uno de los sectores económicos con mayor crecimiento a nivel mundial. Según cifras de la Organización Mundial de Turismo, durante el año 2019 se registraron cerca de 1.500 millones de llegadas de turistas internacionales en el mundo. La expectativa estaba centrada en un incremento para el año 2020 del $4 \%$ con respecto al año anterior, sin embargo, debido a la pandemia Covid19 esto no ocurrió (UNWTO, 2020). Colombia, durante el año 2019, fue visitada por 4.116.120 turistas que llegaron del extranjero, superando en un 2,7\% más las del año 2018 (Ministerio de Industria, Comercio y Turismo de Colombia, 2019).

La búsqueda de destinos y alojamientos turísticos a través de plataformas de metabúsqueda es fundamental hoy en día para la programación de viaje. Según una encuesta realizada por EyeforTravel a más de 3.000 viajeros de Australia, Canadá, Reino Unido, Latinoamérica y Estados Unidos, se revela que el 94,4\% de los consumidores utiliza los sitios de comparación de precios al menos de forma ocasional para reservar su alojamiento. Dentro de este amplio porcentaje, el 72,5\% asegura que deciden con base en los comentarios que reseñan los alojamientos, porque estos reflejan sin máscaras las experiencias de los huéspedes. Según estudio de Nadal (2017), las personas piensan que la información mostrada en los comentarios online es veraz. Y un $60 \%$ presta más atención a los comentarios que a las estrellas o la descripción del local para decidirse por uno u otro. Esto coloca a los comentarios y calificaciones de los metabuscadores de viajes en un lugar privilegiado dentro del proceso de decisión y reserva de un viaje.

El protagonismo otorgado al usuario en la web está haciendo que su opinión se convierta en pilar fundamental de las nuevas estructuras administrativas, y esto ha sido un elemento que ha llevado a fortalecer las capacidades de servicio o habilidades blandas de los empleados de las organizaciones para que se pueda incidir de manera positiva en la experiencia del cliente.

Actualmente existen escenarios virtuales en donde se comparten las experiencias de los viajeros y huéspedes como Booking o Tripadvisor, que han generado nuevas maneras de comunicar y empoderar la opinión del usuario, hasta "crear una especie de comunicación epidemiológica" que ha cambiado la forma de influenciar, pues un porcentaje importante de turistas cree en los comentarios y opiniones de sus pares, es decir, "personas de características similares a ellos mismos, más que en la publicidad generada por las empresas a través de los medios tradicionales" (Hernández \& Mariottini, 2016, p.151).

Según estudio de cibergéneros realizado por Sanmartín y González (2013), se están catalogando las reseñas, comentarios y opiniones hoteleras como un "género emergente" en la comunicación turística actual (Piccioni 2014), en donde la interpersonalidad asume gran importancia (Mapelli, 2008; Suau-Jiménez 2012).

Expresa Calvi (2010), que es evidente la función desenmascaradora de estas tribunas virtuales (Dann, 2007), que ofrecen informaciones detalladas, exentas de propósitos comerciales, sin ocultar decepciones y hasta desilusiones. Estas investigaciones se han convertido en fuentes importantes y valoradas al entender que las clasificaciones generadas por la opinión de los internautas inciden de manera significativa en la decisión de los clientes potenciales (Vermeulen \& Seegers, 2009).

Según lo expresan Kernbach y Schutte (2005), el desarrollo de las habilidades blandas en los empleados se ha convertido en el centro de la industria hotelera. Estas habilidades deben manifestarse a través de la satisfacción al cliente, generando experiencias innovadoras y haciendo énfasis en todo momento en el servicio, además de ofrecer un producto de excelente calidad. Existe, por tanto, la expectativa y la necesidad de contar con personal educado en rasgos como la flexibilidad, la conciencia multicultural y el pensamiento disruptivo, integridad y cordialidad.

La honestidad y el hacer lo correcto deben ser un requisito, las anteriores son claramente habilidades blandas, que tienen que estar implícitas en la personalidad y comportamiento del empleado. Es por este motivo que el hotel, desde su área de contratación, debe prestar atención en las habilidades blandas para lograr un equipo de trabajo que se adapte a los valores y la misión de la línea hotelera. 
Para el hotel objeto del estudio, las cuatro (4) habilidades blandas que se ven reflejadas en sus empleados y que se consideran esenciales son el trabajo en equipo, la relación con el cliente, el servicio al cliente y la integridad. Tobar (2012) define las mencionadas habilidades blandas como destrezas subjetivas e intangibles, y añade otras como el liderazgo, la comunicación fluida, la tranquilidad para manejar los momentos de presión en el trabajo entre las más destacadas.

Esta indagación de literatura existente en torno a ranking en metabuscadores y habilidades blandas, nos ha llevado a plantearnos las siguientes preguntas, que hemos adoptado como guía para realizar la presente investigación: ¿En qué medida los comentarios y opiniones expresados abiertamente en plataformas de turismo y metabuscadores están haciendo replantear la importancia de las soft skills (habilidades blandas), en los trabajadores del sector turístico y hotelero? ¿Cómo suelen responder los gerentes y empleados de los establecimientos evaluados a las críticas? ¿Si responden, qué tipo de respuestas ofrecen? ¿Se deberían implementar capacitaciones para mejorar y potenciar las habilidades blandas de sus empleados para mejorar la experiencia del usuario y que esto se refleje en mejores comentarios? Estas reflexiones nos llevan a plantear un análisis cualitativo y cuantitativo de las experiencias del cliente presentes en un corpus de reseñas hoteleras que permita responder a las cuestiones planteadas.

El hotel objeto de estudio, perteneciente a una cadena hotelera internacional y ubicado en la ciudad de Medellín, es reconocido por su infraestructura y sus servicios de calidad y atención al cliente; el hotel prepara a su personal para prestar un servicio completo fortaleciendo y enseñando las habilidades blandas en diferente áreas como solución de problemas complejos, pensamiento crítico, creatividad, gestión de personal, coordinación con los diferentes agentes participantes, inteligencia emocional, toma de decisiones, orientación al servicio, negociación, flexibilidad cognitiva, las cuales representan algunas de las habilidades blandas más importantes en el mundo laboral.

Según lo indica el estudio sectorial e informe de tendencias en la industria del turismo, de Moving Forward by WAM, "la aceleración de los cambios demanda habilidades blandas y talento adecuado a la necesidad real del mercado. En una industria turística cada vez más inmersa en la transformación digital, en donde la opinión del usuario expresada en redes sociales y plataformas turísticas es resaltada, las empresas del sector que buscan nuevos empleados para sus organizaciones van a primar sobre todo aquellas personas que ofrezcan un buen equilibrio entre habilidades hard (duras) y soft (blandas). La importancia del talento humano para la competitividad de las empresas turísticas ha tomado mayor relevancia en los últimos años, debido al creciente avance de la tecnología en diversos ámbitos del turismo, la variable demográfica y generacional, las transformaciones que vienen sufriendo el empleo y la propia industria hotelera y el turismo que tiene que adaptarse a los requerimientos de los nuevos turistas. El crecimiento de la demanda en el sector del turismo ha llevado consigo un aumento significativo de las oportunidades de empleo en todos los niveles. Un número creciente de destinos en todo el mundo están dispuestos a convertir el turismo en un impulsor clave del progreso socioeconómico.

Una fuerza de trabajo que cuente con ciertas competencias claves fortalecerá la capacidad de su empleador para competir. A su vez, estas competencias brindan al trabajador mayores oportunidades de desarrollo y una mejor calidad de vida. A los trabajadores con buenas habilidades claves les resulta más fácil adquirir destrezas más sofisticadas, para así conseguir mejores empleos y salarios más altos (Carnevale, Gainer, \& Meltzer, 1990).

Se pretende entonces, como objetivo general, determinar la relación entre habilidades blandas de los empleados de un hotel y su impacto en las reseñas positivas y negativas en un metabuscador.

\section{METOdOLOGÍA}

Con la implementación y desarrollo de los procedimientos y metodología propuestos en el artículo, se espera brindar una realidad específica del impacto de las habilidades blandas en la experiencia positiva del usuario en el hotel, lo que debe reflejarse en los comentarios positivos y/o negativos de la plataforma de metabúsqueda. 
Tipo de investigación

Se plantea una investigación cuantitativa, en la recolección de datos y presentación de los resultados se utilizaron técnicas estadísticas para determinar la muestra.

\section{Nivel de la investigación}

La investigación posee un nivel tipo descriptiva - correlacional, que consiste en la medición de la relación existente entre dos variables de estudio, donde la variable independiente "reseñas de los usuarios" genera cambios o incide en la variable dependiente "soft skills".

\section{Diseño de la investigación} variables.

El diseño es No experimental, porque el investigador no realizó ninguna acción intencional sobre las

Transversal, porque la información del objeto de estudio (población) se obtuvo una única vez en un momento determinado.

Descriptiva, según Hernández (2017), “la investigación descriptiva reseña las características de un fenómeno existente".

Correlacional, porque examina la relación entre variables o resultados de variables; proporcionando indicios o predecir un resultado de la relación que podría existir entre dos o más elementos.

\section{Población}

La población del estudio está representada en mil doscientas cuarenta y cuatro (1.244), opiniones que fueron la base para otorgar la calificación del hotel objeto de estudio, en la plataforma de metabúsqueda utilizada.

Booking es un sitio web que proporciona reseñas de contenido relacionado con viajes. También incluye foros de viajeros. Los servicios del sitio web son gratuitos y son los usuarios quienes proporcionan la mayor parte del contenido. El sitio web se financia con publicidad. Se han convertido en los más importantes referentes a nivel mundial, en el momento de realizar reservas hoteleras, pues de acuerdo a los comentarios de los huéspedes, los posibles usuarios toman las decisiones o no de compra.

Muestra

Para la muestra se tomaron doscientos noventa y seis (296) referencias de la mencionada plataforma, teniendo en cuenta la fórmula para calcular el tamaño de muestra:

$$
n=\frac{N \times Z_{a}{ }^{2} \times p \times q}{d^{2} \times(N-1)+Z_{a}{ }^{2} \times p \times q}
$$

En donde $\mathrm{N}$ = tamaño de la población (1.244) $\mathrm{Z}$ = nivel de confianza (95\%) $\mathrm{P}$ = probabilidad de éxito, o proporción esperada $\mathrm{Q}=$ probabilidad de fracaso $\mathrm{D}$ = precisión (error máximo admisible en términos de proporción $5 \%$.

\section{Procedimiento}

\section{Etapa 1}

Se tomó la muestra correspondiente a doscientos noventa y seis (296) comentarios extraídos del metabuscador de turismo y reservas mediante el programa ParseHub, el cual desarrolla un algoritmo de búsqueda y extracción siguiendo órdenes precisas. Este programa arroja un archivo de tipo .xlsx con todos los datos en forma de texto, correspondientes a los comentarios y opiniones que dan cuenta de la experiencia del usuario en el hotel, en un lapso de treinta y siete (37) meses. 


\section{Etapa 2}

A través del Programa R, en su interfase R Studio y mediante el uso de las librerías Here y Tidyverse, se realizaron las gráficas y la importación de datos desde Excel, filtrando las reseñas a través de tres (3) palabras principales: malo, regular y bueno, las cuales dan cuenta de la calificación de las reseñas. A lo largo de los comentarios se observan las palabras "mal" o "malo" asociado a malas experiencias; "personal" asociado a mal servicio (generalmente); "ruido" asociado a calificaciones regulares o negativas; "cuenta" asociado a descuadres o robos y "bueno y atención” asociado a buenas experiencias.

\section{Etapa 3}

Se determina la relación entre malas, buenas y regulares calificaciones con el desempeño o no de las habilidades blandas en el desempeño de los empleados del hotel.

\section{Resultados}

Mediante la recopilación de los comentarios ordenados por relevancia de Booking, se tomó una muestra estadística con un 95\% de confianza, en donde se analizaron 297 reseñas.

En los principales hallazgos se encontró que dependiendo de las reseñas se repiten ciertas palabras, muchas veces se conjugan en las mismas frases las mismas palabras positivas o negativas, estas palabras fueron filtradas por las más recurrentes y en algunas ocasiones se utilizó un sinónimo de las mismas para facilitar el manejo de datos. Por ejemplo, la palabra "mal" se utilizó como palabra genérica para todos sus sinónimos como "horrible", "terrible", etc. Se utilizó "restaurante" para los comentarios asociados al servicio de restaurante directamente, "bueno" como palabra genérica para "bien' y palabras que expresan un resultado positivo, cabe anotar que "excelente" se utilizó para las calificaciones mejor valoradas. Las palabras se utilizaron en minúscula y sin tildes para poder optimizar el análisis, puesto que las reseñas no fueron corregidas ortográficamente.

El criterio para elegir estas palabras está relacionado también con su número de coincidencias, su relación con las cuatro (4) habilidades blandas detalladas en este artículo y las razones más comunes de insatisfacción en un huésped. Estas cuatro (4) habilidades blandas se construyeron basándose en estudios previos de las habilidades blandas específicas de compañías que planean mejorar su área de reclutamiento.

En la tabla frecuencia que se encuentra más adelante se pueden ver los resultados de las coincidencias por cada palabra y habilidad blanda. Los resultados fueron analizados principalmente para las palabras con mayor número de recurrencias. Se dividió también por años la información, para generar un promedio de las calificaciones de hotel, siendo 2018 el año con mejores calificaciones y 2020 el año con las calificaciones más bajas.

\section{Relación palabras reseñas Vs Soft Skills}




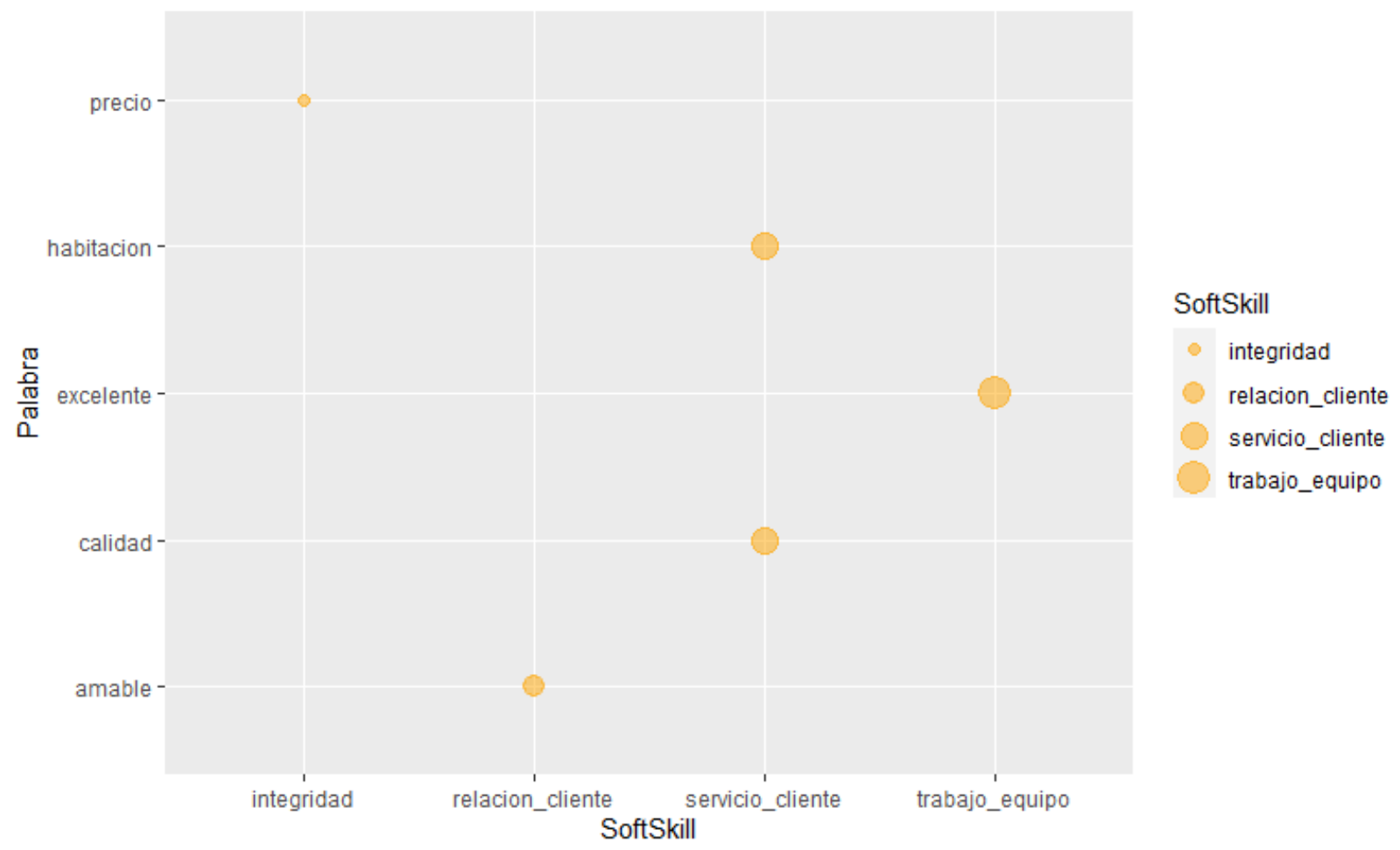

Gráfica 1. Relación palabras reseñas VS Soft SKills. Elaboración propia.

El tamaño de los puntos expresa las coincidencias de la palabra, "excelente" fue la palabra con mayor coincidencias y se relacionó con el trabajo en equipo, ya que una calificación 10 de 10 de Booking requiere que todas las áreas hayan estado conectadas y trabajando en equipo.

Cabe resaltar que la palabra "habitación" se relaciona con el servicio al cliente, asociada con reseñas negativas por un servicio lento o una habitación con falta de aseo. "Amable" hizo parte de la relación que lograban ciertos empleados con los usuarios, en algunas ocasiones se acompañaba la palabra "amable" con el nombre del empleado que dejó una marca positiva en la experiencia del cliente.

Finalmente, el precio se relacionó con la integridad, ya que fueron problemas con el manejo de el dinero o las tarjetas de los clientes que dejaron una mala experiencia en el cliente. 


\section{Rating promedio por año}

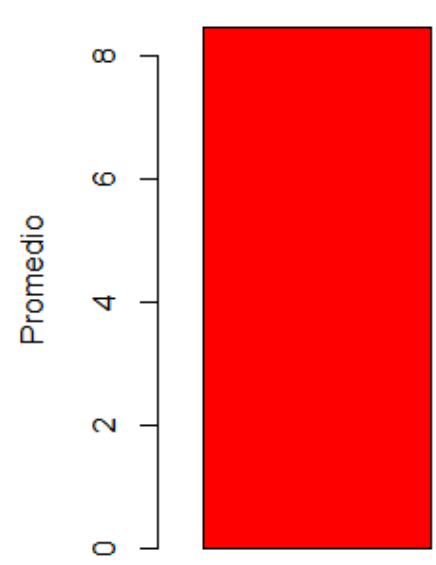

2020

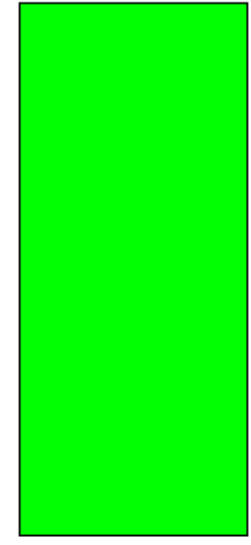

2019

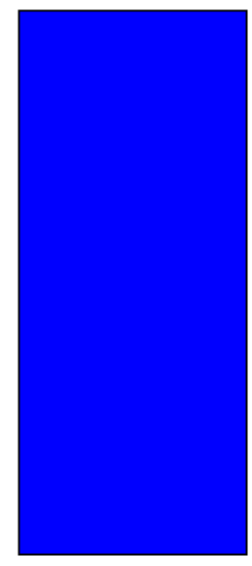

2018

Años

Gráfica 2. Según los años el promedio de calificaciones online. Elaboración propia.

La gráfica muestra el promedio de calificación a través de los tres (3) años analizados en orden ascendente. El 2020 fue el año con mejor calificación y el 2018 fue el año con mejor clasificación según un promedio de sus calificaciones observadas en cada año. Se observan grupos de malas reseñas en el año 2020, con el azul más oscuro, muchas relacionadas al desayuno, al ruido, o a la falta de relación con los empleados del hotel, los usuarios no se sentían atendidos ni generaban una conexión con este.

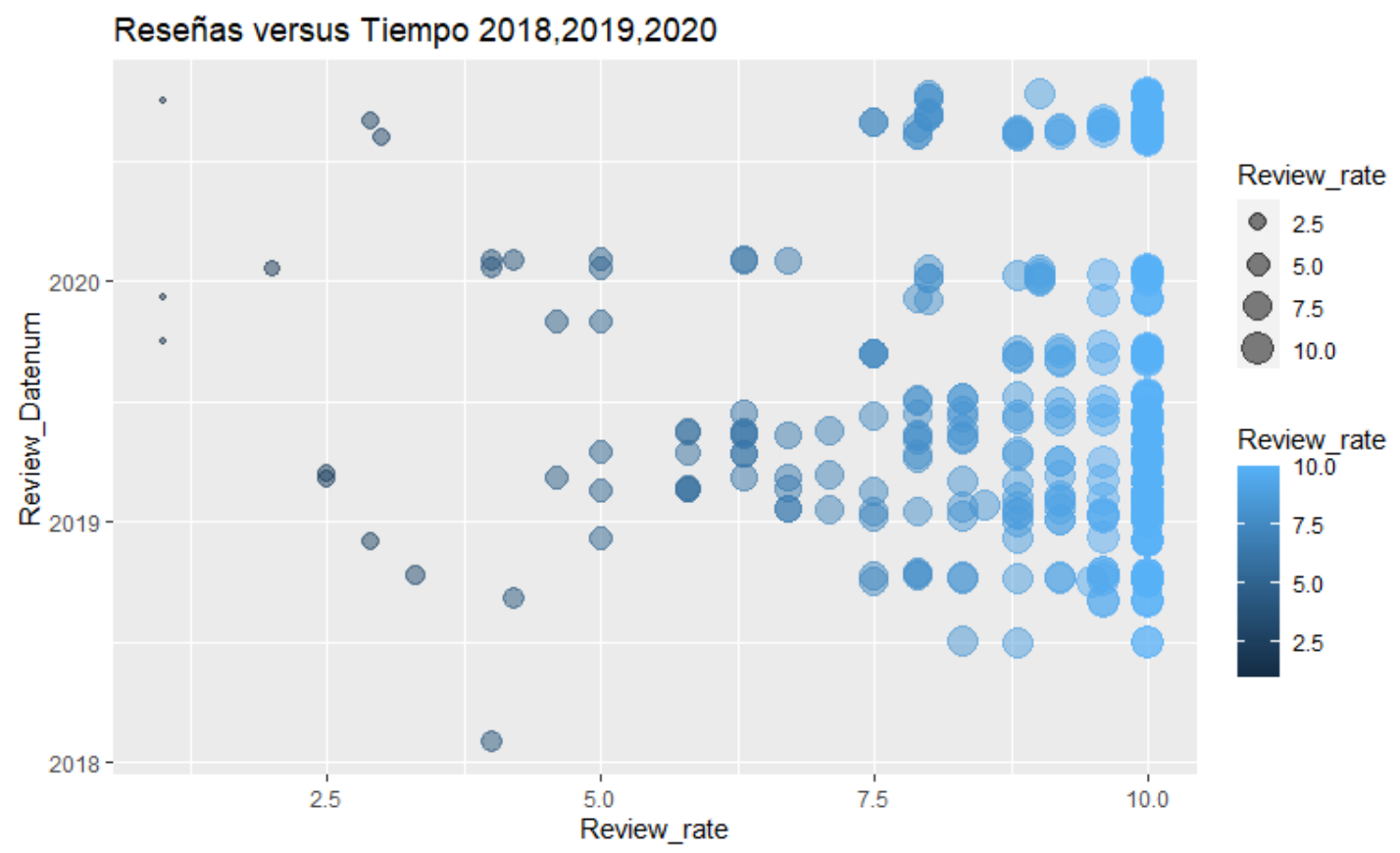

Reseñas Versus tiempo 2018, 2019, 2020

Gráfica 3. Reseñas Versus tiempo 2018, 2019, 2020. Elaboración propia. 
Este es otro análisis gráfico de las reseñas según cada año, de manera individual y ya no como un promedio. Se pueden observar grupos de calificaciones bajas, así como calificaciones malas independientes. En general, las mejores calificaciones vienen por grupos y por fechas similares.

En la siguiente gráfica se ven reflejadas todas las palabras que se identificaron en la muestra estadística de reseñas, se puede ver la frecuencias de las palabras utilizadas, algunas palabras tuvieron solo una coincidencia y otras palabras se utilizaron sinónimos para facilidad de analizar la información. Como se menciona al inicio de los resultados, es necesario resaltar que estas palabras aunque tienden a relacionarse con un nivel de calificación ya sea malo o bueno, en ocasiones son utilizadas en un sentido opuesto. El restaurante, por ejemplo, fue usado en malas y buenas reseñas (Principalmente malas).

\section{Según las calificaciones a través de los años, coincidencia de palabras en las reseñas}

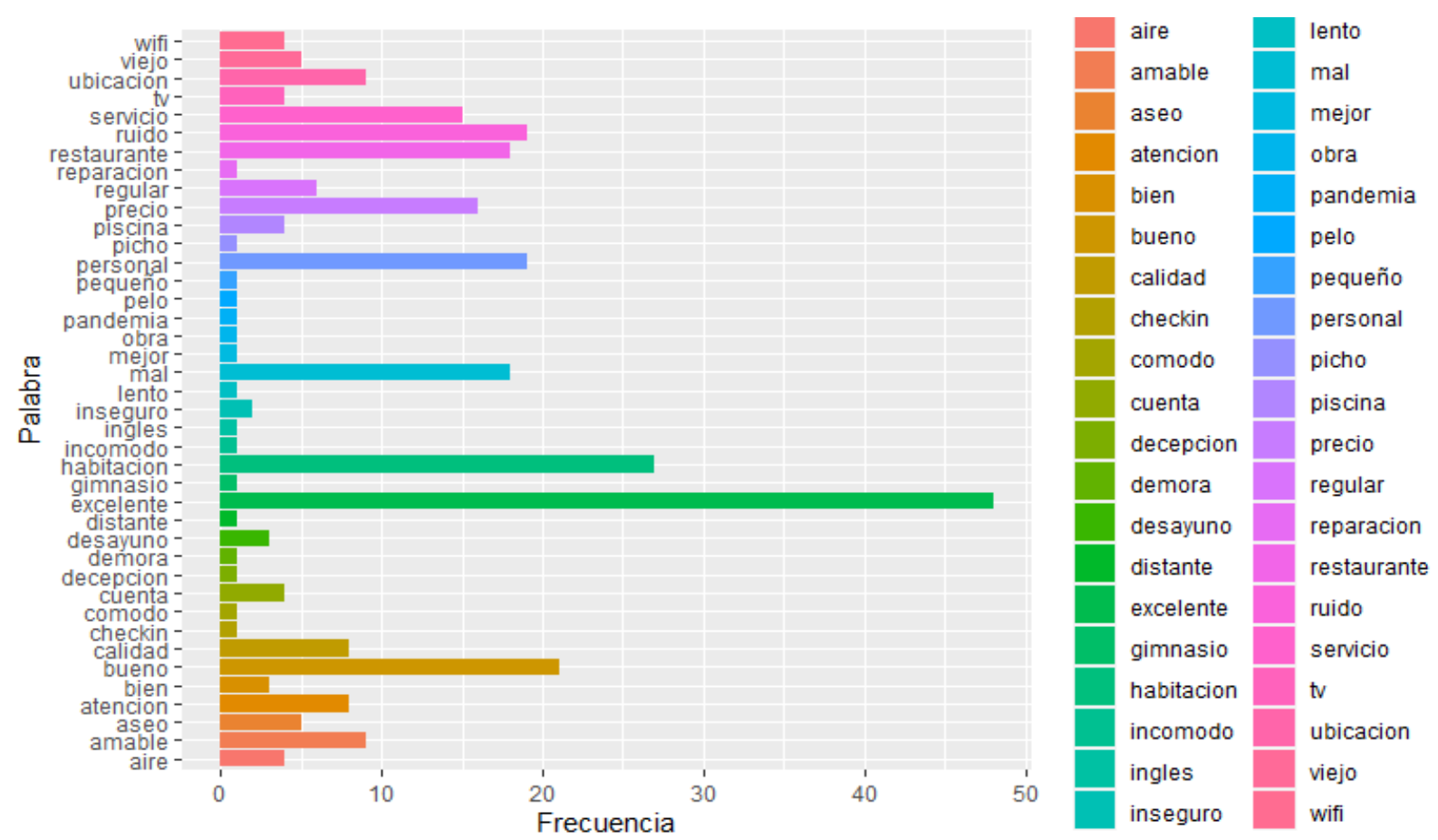

Gráfica 4. Según las calificaciones a través de los años, coincidencia de palabras en las reseñas. Elaboración propia.

En la Gráfica 5, el servicio al cliente fue la habilidad blanda que tuvo más coincidencias con relación a la lista de palabras recurrentes, seguida del trabajo en equipo, la relación con el cliente y por último la integridad. En especial con la integridad no se vieron reflejados comentarios positivos o resaltando la honestidad del personal. 


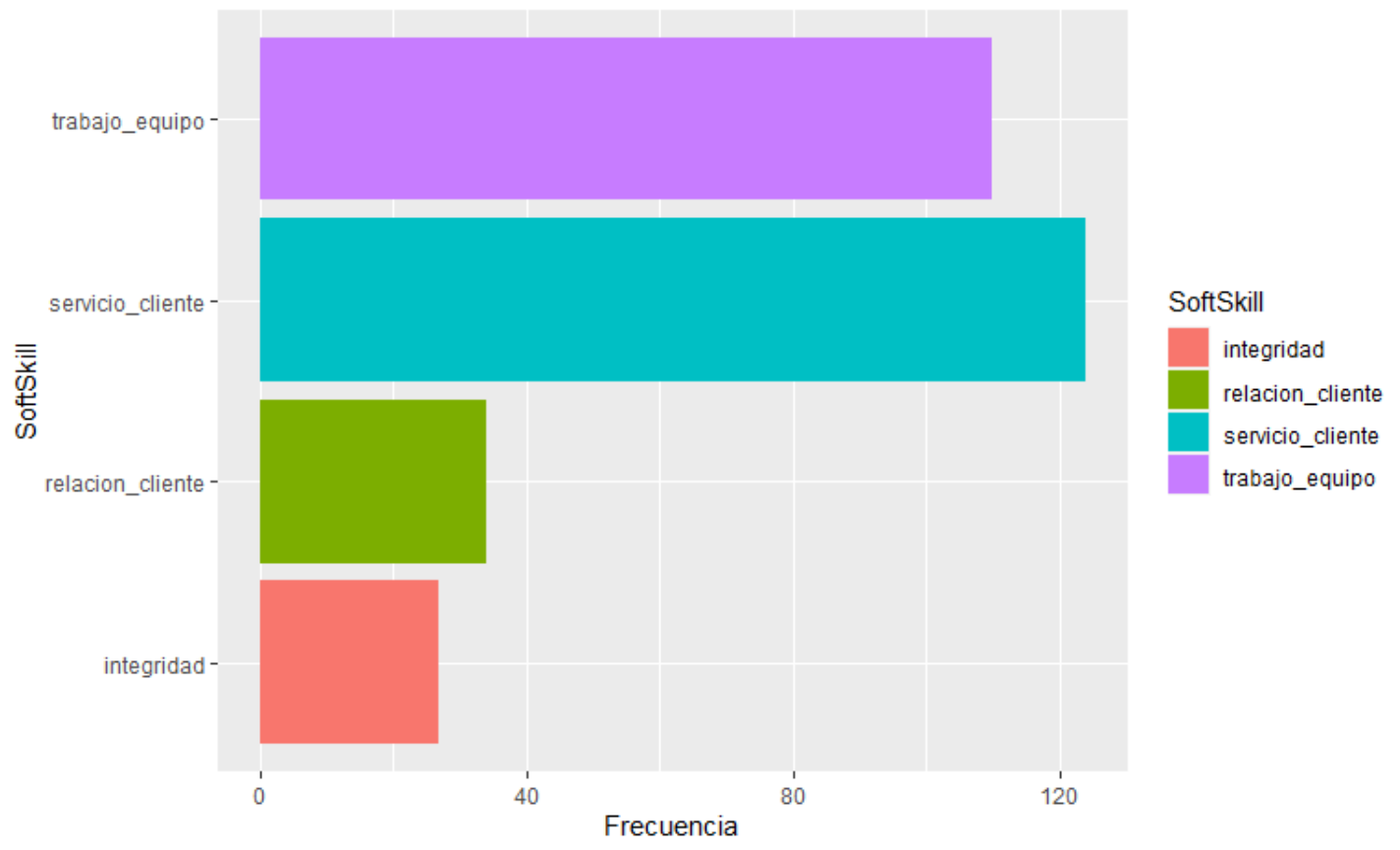

Frecuencia de las soft skills (habilidades blandas)

Gráfica 5. Frecuencia de las soft skills (habilidades blandas). Elaboración propia.

\section{ANÁLISIS DE LOS RESULTADOS}

Este estudio se enfocó en el análisis y la recopilación de datos utilizando conceptos de habilidades blandas, que son familiares para cualquier persona que haya tenido una experiencia usuario-proveedor. Una gran cantidad de reseñas online le hablan al usuario directamente con números, acerca de buenas calificaciones, o con palabras malas y buenas. Muchos hoteles pueden tener sus fortalezas y debilidades claramente detalladas en sus bases de datos de reseñas en línea. Un hotel con malas calificaciones puede ser un hotel que no supo manejar su imagen y relaciones con el cliente y no es necesariamente un mal hotel, pero esto afectará directamente su recepción y popularidad, lo cual es fuente vital para un hotel; si su impopularidad aumenta, las personas lo van a evadir. Se considera que las reseñas deben ser estudiadas teniendo un margen de reserva, ya que muchas veces quien publica una reseña no sabe realmente cómo funciona o cómo se califica según la página web. Esto puede generar que se den mejores reseñas, con comentarios malos o malas reseñas con comentarios buenos, por no saber cual es el nivel cuantitativo para calificar esta reseña y esto varía en cada plataforma web. En Booking en particular la mejor calificación es 10, pero se encontraron calificaciones de 10 con relatos sobre experiencias desagradables.

El estudio en su parte teórica recopiló información para sustentar y definir las habilidades blandas en conceptos, con los valores y las experticias que una cadena hotelera debería proyectar, por medio de reseñas en línea en una base de datos pública. El origen de los documentos corresponde a diferentes bases académicas especializadas en el área hotelera; en general, los artículos seleccionados son de carácter científico, afines a la temática estudiada. Esta información se registró en un documento Excel, durante ocho (8) semanas.

Según información extraída de la página web del hotel objeto de estudio, se tienen definidos sus valores: respeto, compromiso, integridad, cooperación y proactividad. Para la situación problemática a trabajar se decidió que el enfoque fueran la integridad y la cooperación, ya que estos dos valores son los pilares que fortalecen una ética de trabajo imperante, para que una línea hotelera de lujo sea capaz de brindar una experiencia completa a sus usuarios, dejando un recuerdo inolvidable que los haga volver una y otra vez.

Según Robles (2012), en su artículo "Executive Perceptions of the Top 10 Soft Skills Needed in Today's Workplace", en los primeros diez lugares de las habilidades blandas, según ejecutivos entrevistados en el artículo, están listadas las mencionadas anteriormente, aunque no es el principal enfoque de este estudio, pues se considera que falta mucha información para llegar a proponer sugerencias de 
capacitaciones para las grandes cadenas hoteleras, acerca de cómo mejorar sus relaciones con los clientes. Se considera este artículo como un punto válido de referencia, que puede tomar una compañía o cadena hotelera para identificar qué tipo de cualidades debe buscar en su área de contratación.

La habilidad blanda más recurrente en este artículo fue el servicio al cliente, pero se evidenció que el trabajo en equipo es el causante directo de las mejores reseñas, porque una experiencia en una estadía tiene muchos escenarios y relaciones que se generan entre el usuario, el hotel y el personal, por lo cual las áreas deben estar perfectamente engranadas para que el hotel en su totalidad tenga un buen desempeño. Una experiencia positiva completa no es posible sin un equipo de trabajo, empezando con el recepcionista, el personal de aseo o personal de la atención primaria del servicio al cliente, hasta los guardias de seguridad que no tienen una relación estrecha con el usuario, pero pueden generar puntos extras si se comportan de manera cordial, haciendo sentir a los huéspedes que están en un ambiente de cooperación y seguridad, en donde la integridad es una regla tácita, para que aquellos que se hospeden puedan dejar su equipaje y disfrutar de su estadía sin preocupaciones.

En la primera gráfica, en donde se relacionaron las soft skill frente a algunas de las palabras más relevantes, se omitieron muchas palabras para permitir un análisis claro de la relación que se buscó con este artículo. Se considera las habilidades blandas como algo que tiene un impacto directo en las experiencias del cliente, y no solo en lo que piensa sobre el hotel sino en lo que siente por el hotel y su experiencia de hospedaje, la que se convierte en una experiencia íntima, donde el huésped fácilmente se puede ver vulnerado. Por eso la opinión del huésped reflejada en las reseñas que cuentan sus experiencias no solo evidencia lo que observaron y analizaron sobre su experiencia, sino lo que sintieron y si quieren volver a experimentarlo. Esto genera lealtad y apego a un hotel, haciendo de un cliente de una vez un usuario recurrente que mantenga una y otra vez la reputación del hotel en lo alto. Es útil el conocimiento de estas palabras recurrentes en las reseñas, para identificar los puntos de falla o las fortalezas del hotel; "mal" y "personal" muchas veces estaban en la misma reseña, por lo cual concluimos que un punto a mejorar es la relación con el cliente; "cuenta” estuvo relacionado con problemas de integridad, transacciones incorrectas, mostrando lo que a nivel de habilidades blandas se considera como falta en la integridad. Mientras que "excelente" muestra un trabajo en equipo y un buen clima laboral, y "bueno" muestra ya sea la combinación completa o parcial de las cuatro habilidades blandas. Una palabra que resultó del análisis de datos fue "precio", que lo atribuimos a la falta de trabajo en equipo y falta de buenos proceso de contratación, que permitan contratar empleados confiables, ya que la integridad es una habilidad que muchas veces viene desde la educación en el hogar.

Considerando la tercera gráfica de las reseñas frente al tiempo, podemos ver que las reseñas tienden a agruparse, lo que evidencia que no solo son casualidades, sino que existen problemas o fortalezas de fondo que causan estos efectos. El resultado de esta segunda gráfica de reseñas frente al tiempo nos enseña un mapa temporal de la experiencia vivida por el usuario en la fecha publicada. Los colores más claros y los puntos más gruesos reflejan las mejores calificaciones. Como se prueba en la gráfica dos (2) de los ‘Rating promedio por año’ se ve una agrupación de malas reseñas en el 2020. Aunque la diferencia no es muy notoria, el 2018 muestra no solo mayor concentración de buenas reseñas, sino un menor número de reseñas negativas.

Es necesario mencionar que el análisis exitoso de base de datos requiere de una buena estructura de software para lograr conseguir un volumen de relevantes en un lapso de tiempo razonable. En esta primera incursión en el análisis de datos, en la cuarta gráfica titulada 'Según las calificaciones a través de los años', se compiló toda la información sobre las coincidencias de las palabras: 296 datos en los que se buscó recoger el menor número de palabras sin omitir información relevante para tener una impresión general de lo que se piensa de este hotel, que se analizó en línea. Este hotel del cual se estudiaron sus reseñas, tiene ubicación en Medellín, por eso algunas palabras aunque no tienen mayor relevancia, como lo son "aire" o "piscina", tienen un impacto directo con la experiencia del cliente, se escogió utilizar estas palabras, ya que eran lo que principalmente define la reseña. El aire se utilizó en algunas ocasiones, porque las reseñas reflejaban quejas sobre el aire acondicionado siendo "viejo", y que tenía cierta hora en donde se apagaba. En general, las experiencias con el 'aire’ o ‘viejo' fueron negativas. Por otro lado, la piscina se resaltó como una fortaleza, ya que se habló de su limpieza. Aunque estas dos palabras en particular no tienen una relación directa con ninguna habilidad blanda, se consideran componentes de un buen servicio al cliente. Las dos palabras con más coincidencias, 'excelente' y 'habitación', tienen en este artículo un significado 
mayormente opuesto. Las habitaciones y el servicio a la habitación, que se relacionó con la habilidad blanda de servicio al cliente, tenía comentarios negativos sobre su falta de coordinación. Mientras que la palabra 'excelente' se relacionó con experiencias en donde muchas veces no existen comentarios, solo muestras de total satisfacción en donde no había nada adicional que comentar.

En la gráfica cinco (5), 'frecuencia de las soft skills' el dato menos recurrente es el dato que de hecho muestra los puntos críticos del hotel, que se deberían considerar y tratar de inmediato; la integridad se puede relacionar con temas de fraude, cargos no aprobados, pequeñas o grandes pérdidas de objetos personales. A pesar de que no fueron muchas las reseñas que mencionan algún tema de integridad, las reseñas relacionadas con palabras como 'precio' o 'inseguridad' son muestras de que el hotel debe considerar replantear su proceso de reclutamiento, en el que se logren construir filtros que prueben la honestidad, la sinceridad y el manejo profesional de los empleados, no solo en cuanto a los objetos de los demás. sino en cuanto a las cuentas, para tener la capacidad de llevarlas claras y transparentes. La honestidad y la sinceridad, a pesar de que pueden ser reforzadas con entrenamiento, son habilidades blandas que se cultivan desde el hogar; es por eso que recalcamos la importancia de un proceso de selección que tenga en cuenta las cuatro (4) habilidades blandas mencionadas en este artículo.

Por último, se definieron las diferencias entre relación con el cliente y el servicio al cliente, pues pueden tener significados similares, pero en este artículo se evidencian los puntos de divergencia entre estas dos habilidades blandas. El servicio al cliente consiste en el deber que tiene el personal de proveer al usuario todas las resoluciones posibles a las diferentes eventualidades y en el tiempo racional, esto se ve reflejado en cuanto calidad de servicio al cuarto, calidad y efectividad en los tiempos del restaurante, en el proceso de check-in, entre otros, entonces el servicio al cliente en este estudio podría tener una unidad de valor como la calidad ofrecida del servicio. Mientras que la relación con el cliente se enfoca en la empatía que los empleados y la línea hotelera logran obtener con el cliente, personalizando la experiencia, realizando cumplidos adecuados y haciendo sentir al cliente acogido y seguro en su tiempo de hospedaje.

Teniendo en cuenta que las reseñas fueron extraídas de Booking, las calificaciones fueron tomadas en un rango de 1 a 10 estrellas. Según esta gráfica, se decide considerar al trabajo en equipo como la habilidad blanda con más impacto de las cuatro tratadas. Consideramos la razón de esto que cuando la habilidad blanda falla, y no hay trabajo en equipo, todas las otras fallan también y la calificación se desmorona en conjunto, ya que si no hay relación entre las áreas no se podrá presentar un servicio de calidad, íntegro, y mucho menos se podrá generar una relación positiva con el cliente. Este trabajo en equipo también se ve reflejado positivamente en una reseña que logró las cinco 10 estrellas. Se enfatiza en que es solo la combinación de todas las habilidades blandas mencionadas, lo que permite generar una experiencia positiva en el cliente, ya que creemos que una experiencia en el hotel debe ser segura, consistente en todas sus áreas, debe dejar una marca personal en el huésped y deben cumplir todas sus necesidades.

La organización en una línea hotelera específicamente situada en Medellín, que cuenta con 139 habitaciones y un auditorio con una capacidad de albergar 1.250 personas, hace necesario que el nivel gerencial tenga un enfoque en la teoría de solución de problemas empresariales y posea un equipo moderno y flexible, que esté siempre desempeñándose al máximo, para que en conjunto con la automatización de su infraestructura puedan lograr manejar un alto volumen de usuarios. Las reseñas analizadas hablan particularmente de la lentitud del servicio y de la falta de organización como en el ruido del cual muchos usuarios se quejan.

\section{CONCLUSIONES}

Como se puede observar, varios sitios web se especializan en permitir a los usuarios publicar reseñas en línea. En particular los sitios de los que se extrajeron las reseñas se consideraron por el volumen de reseñas y el fácil acceso a las mismas. Nos basamos principalmente en la primicia de que las reseñas hoy en día son consideradas una de las principales motivaciones a la hora de la selección del hotel, impactando directamente en las decisiones de un consumidor para optar por un hotel u otro, en un mercado altamente competitivo. 
A pesar de que este es solo un estudio preliminar, tenemos en cuenta una desviación posible en cuanto a la veracidad de las reseñas, aunque se considera que esta puede no ser tan significativa. En las reseñas de Booking se debe contar con un número de reserva para poder publicar la experiencia.

Según los estudios observados y la investigación que se llevó a cabo, se pretende ser enfáticos en que no existe una habilidad blanda más importante que otra sino que la diferencia se evidencia en el conjunto de las habilidades aplicadas en el momento adecuado.

Se considera que, además de las habilidades blandas inherentes de cada empleado, para que tengan éxito, depende también de la capacidad de los líderes de la línea hotelera para generar una relación estrecha entre el empleado y la compañía. Es importante notar que las capacitaciones continuas, en áreas especializadas de las habilidades blandas son necesarias para mantener un equipo de trabajo con el mejor desempeño.

Las actividades de integración donde se refuerce públicamente los valores y la misión de la empresa, generando metas para los empleados, que promuevan cultivar y mantener las habilidades blandas, en conjunto con el resto de habilidades duras.

Es importante notar que fuera de las actividades de refuerzo que puede promover la empresa para aumentar la lealtad, se considera que el área de recursos humanos debe tener un proceso estratégico de selección, en donde se pueda encontrar un equipo de trabajo que logre apegarse a los valores que son más importantes para la empresa, ya que muchas habilidades blandas como la honestidad, que viene a encajar con la soft kill de la integridad no es algo que se pueda aprender en entrenamiento, sino que se debe haber enseñado desde los valores del hogar. Es por eso que no solo se deben buscar empleados con capacidades desarrolladas en cuanto a las habilidades prácticas o duras, sino con valores personales de alta calidad.

Como fue planteado en el objetivo general de este estudio, es importante que los hoteles replanteen no solo sus procesos de reclutamiento, sino se vuelven constantes en diseñar un plan de capacitación que presente escenarios posibles con diferentes tipos de clientes, en donde el empleado siempre sepa evaluar correctamente cada situación y poner las necesidades del huésped sobre cualquier otra.

Enseñarle a los empleados a manejar cada situación no solo con una respuesta, sino con un proceso elaborado, en donde se reflejen las habilidades personales, generando una cadena de respuestas positivas con el cliente. Estos escenarios de entrenamiento permitirán al personal estar preparado para las diferentes eventualidades que se puedan presentar, enseñando los procesos y metodologías para que estos sirvan como potenciadores de las habilidades blandas, que el área de reclutamiento debe encargarse de agregar a su talento humano en el momento de contratarlos.

Cabe anotar la importancia de la opinión del cliente por medio de plataformas tecnológicas; debe volverse primordial ofrecer una respuesta efectiva a estos comentarios, no solo brindando un comentario de aliento o de disculpas por una mala experiencia o respondiendo los mejores comentarios, como es usual. La gerencia del hotel y su personal encargado del servicio al cliente post venta deben encargarse de hacer seguimiento a las diferentes inquietudes y quejas de los clientes, para lograr resolver estos problemas de raíz, dando soluciones efectivas que permitan aliviar el estrés o los daños generados al usuario.

Se busca enfocarse en el tema de hacer seguimiento a las inquietudes, ya que muchas veces se considera que una respuesta clara es la solución, pero además de esto, una inquietud se debe considerar como una investigación independientemente de su gravedad; las compañías o cadenas hoteleras deben contar con el personal y los recursos para responder a las malas reseñas y buscar mejorar esa experiencia, ya sea mediante descuentos, promociones para motivar al cliente a volver, o por medio de reembolsos y recuperación si se causaron daños en la integridad del material del usuario.

Adicional al precio del hotel, las reseñas son un factor definitivo a la hora de escoger entre una opción u otra. La breve investigación que realiza una persona a la hora de escoger un hotel, sea por turismo o su trabajo, consiste en leer las experiencias que otros usuarios tuvieron con el hotel, para a partir de eso tomar una decisión prevenida de si el dinero invertido por esta experiencia de hospedarse en el hotel va a tener una retribución justa al costo y va a llegar a un lugar donde se siente cómodo, seguro y además puede contar a los demás que estuvo en el hotel que tan buenas reseñas tiene y compartirlo en las redes. Se 
considera que la relevancia de un hotel en la web, hoy en día es una carta presentación para la mayoría de las personas, y puede influenciar definitivamente la inversión de un usuario en un hotel en particular.

Por otro lado, se busca recalcar que para hoteles con gran volumen de huéspedes, como el analizado en este artículo, se genera un gran número de reseñas, por lo cual para realizar un análisis significativo, en un tiempo que permita realmente lograr conclusiones claras, se requiere conocimiento en el manejo de bases de datos, para poder conjugar de manera lógica los comentarios en línea, pues en primera instancia -por ejemplo- la palabra 'viejo' puede no tener ninguna relevancia en cuanto a las habilidades blandas, pero cuando se logra encontrar las coincidencias suficientes y concatenar el valor de la reseña con las palabras usadas, se pueden ver reflejados los mismos sentimientos y opiniones de un cliente a nivel personal y a nivel racional con el hotel que visitó.

\section{REFERENCIAS BIBLIOGRÁFICAS}

Ascolese, G., \& Llantada, J. (2018). Estudio sectorial e informe de tendencias en la industria del turismo, de Moving Forward by WAM. Recuperado de https://www.segittur.es/wp-

content/uploads/2019/10/Estudio-sectorial-e-informe-de-tendencias-en-la-industria-del-turismo1.pdf

Anderson, C., \& Han, S. (2016). Hotel performance impact of social engaging with costumers. Cornell Hospitality Report, 16(10), 1-11.

Booking. (2020). Recuperado de: https://www.booking.com/reviews/co/hotel/dann-carltonmedellin.es.html?aid=357029\&label=yho748jc-

1DCAooMkIVZGFubi1jYXJsdGguLW1IZGVsbGluSApYA2gyiAEBmAEKUAEHyAEN2AED6AEB-

AECiAIBqAIDUAKet438BCACAdICJGJIZDMzOWE1LWFmYzEtNDg2My05NGZmLTcoYzl1YjhmYTUoZd gCBOACAQ\&sid=4edgafb1d59df38468fgc218eedo3f32\&r_lang=es\&customer_type=total\&order=s core_asc

Bisquerra, R. (2006). Orientación psicopedagógica y educación emocional. Recuperado de http://dadun.unav.edu/bitstream/10171/9208/1/Ea.pdf

Carnevale, A., Gainer, L., \& Meltzer, A. (1990). The Essential Skills Employers Want. San Francisco: Jossey-Bass Inc. Publishers

Calvi, M. (2010). Los géneros discursivos en la lengua del turismo: una propuesta de clasificación. Ibérica: Revista de la Asociación Europea de Lenguas para Fines Específicos (AELFE), 19, 9-32.

Commission for Business and Economic Education [PCBEE]. (2000). This we believe about teaching soft skills: Human relations, self-management, and workplace enhancement. Policy Statement 67. Recuperado de http://www.nbea.org/curriculum/no67.html

Crowston, K., \& Williams, M. (2000). Reproduced and emergent genres of communication on the WorldWide Web. Information Society, 16(3), 201-215.

Dann, G. (2007). Revisiting the language of tourism: What tourist and tourees are saying. En: de Stasio, Clotilde y Palusci, Oriana (eds.), The Language of Tourism. Turismo e mediazione. Milán: Unicopli, 1532.

Educamericas. (2019). La importancia de cultivar la ética profesional. Recuperado de http://www.educamericas.com/articulos/reportajes/laimportancia-de-cultivar-la-etica-profesiona

Hernández, M., \& Mariottini, L. (2016). TripAdvisor y actos de habla. Delimitaciones teóricas y propuestas metodológicas para el análisis de las estrategias de atenuación de los actos directivos. Pragmática Sociocultural, 4(2), 149-181. 
Hernández, R. (2017). Metodología de la investigación.

https://www.uv.mx/personal/cbustamante/files/2011/06/Metodologia-de-la-

Investigaci\%C3\%83\%C2\%B3n_Sampieri.pdf

IBM. (2019). The enterprise guide to closing the skills gap. https://www.ibm.com/thought-leadership/institutebusiness-value/report/closing-skills-gap

Kernbach, S., \& Schutte, N. (2005). The Impact of Service Provider Emotional Intelligence on Customer Satisfaction. Journal of Services Marketing, 19(7), 438-444

Mayzlin, D., Dove, Y., \& Chevalier, J.(2012). Who Gave That Hotel Five Stars? The Concierge. Harvard Business Review, Edición septiembre. https://hbr.org/2012/og/who-gave-that-hotel-five-stars-the-concierge

Ministerio de Industria, Comercio y Turismo de Colombia. (2019). Informe turismo 2019 Colombia. Recuperado de https://www.mincit.gov.co/getattachment/estudios-economicos/estadisticas-einformes/informes-de-turismo/2019/diciembre/oee-turismo-enero-diciembre-12-03-202029abr2020.pdf.aspx

Nadal, P. (2017). ¿Cómo sabe TripAdvisor que no soy un mentiroso? El País, 12- 05-2017. Recuperado de https://elpais.com/elpais/2017/05/12/paco_nadal/1494590024_407445.html

Organización Internacional de Turismo. (2019). Recuperado de https://www.unwto.org/es/el-turismomundial-consolida-su-crecimiento-en-2019

Piccioni, S. (2014). Cortesía y lenguas de especialidad entre lo local y lo global: el caso de las reseñas de hoteles en español e inglés. Normas, 4, 93-116.

Puga, J. (2008). Competencias Directivas En Escenarios Globales. Estudios Gerenciales, 24(109), 87-103. https://doi.org/10.1016/S0123-5923(08)70054-8

Tobar, R. (2012). Desarrollo de destrezas, competencias y actitud. Bogotá: ECOE Ediciones.

Suau-Jiménez, Francisca (2012). El turista 2.0 como receptor de la promoción turística: estrategias lingüísticas e importancia de su estudio. Pasos. Revista de turismo y patrimonio cultural, 10(4), 143-154.

Vilardi, R. (2019). Estudio sectorial e informe de tendencias en la industria del turismo. Recuperado de https://www.wearemarketing.com/es/blog/eventos-ux.html

Vermeulen, I., \& Seegers, D. (2008). Tried and tested: The impact of online hotel reviews on consumer consideration. 